\title{
Analytical calculation of the deflection of an externally statically indeterminate lattice truss
}

\author{
Mikhail Kirsanov ${ }^{1, *}$, Evgeny Komerzan ${ }^{1}$, and Olesya Sviridenko ${ }^{1}$ \\ ${ }^{1}$ National Research University "Moscow Power Engineering Institute", Krasnokazarmennaya 14, \\ Moscow, 111250, Russia
}

\begin{abstract}
A scheme of a statically definable truss with additional supports is proposed. Derive formulas for the dependence of the deflection of the truss against the number of panels for three types of symmetrical loads. It is shown that for definite numbers of panels the determinant of the system of equations for the equilibrium of nodes degenerates. This indicates an instant changeability of the structure. To generalize particular solutions to an arbitrary number of panels, the induction method is applied. For this purpose, in the computer mathematics system Maple linear recurrence equations are constructed for the terms of a sequence of coefficients from individual solutions. The graphs of the dependences obtained indicate a nonmonotonic character of the solutions found and the possibility of optimizing the design by choosing the number of panels.
\end{abstract}

\section{Intoduction}

Calculation of the deflection of truss is usually performed numerically in specialized packages based on the finite element method [1-5]. Less common are analytical solutions that use finite-form formulas. In [7-9], solutions for plane symmetric trusses were obtained by induction. More complicated solutions in the form of formulas for regular spatial trusses were obtained in [10-12]. The object of inductive analysis in [13] is a pile foundation with a regular arrangement of piles. Analytic solutions of the problem of a planar truss with allowance for creep of the material are given in [14]. An overview of some other works in this direction was made by D. Tinkov in [15]. Analytical solutions can be used to solve and test solutions of optimization problems [16-19].

\section{Truss and Methods}

A planar truss (Fig. 1) with a height of $4 h$ has a span length of $L=4 a(n+1)$ in which $n$ number of panels $2 a$ in length in half span. The truss contains $m=8 n+22$ rods, including five support rods. Three bearing supports correspond to three support rods. The fixed hinge support (at the right end of the truss) is modeled by two rods. The lateral rods in the upper belt are of length $c=\sqrt{a^{2}+h^{2}}$.

\footnotetext{
* Corresponding author: c216@ya.ru
} 


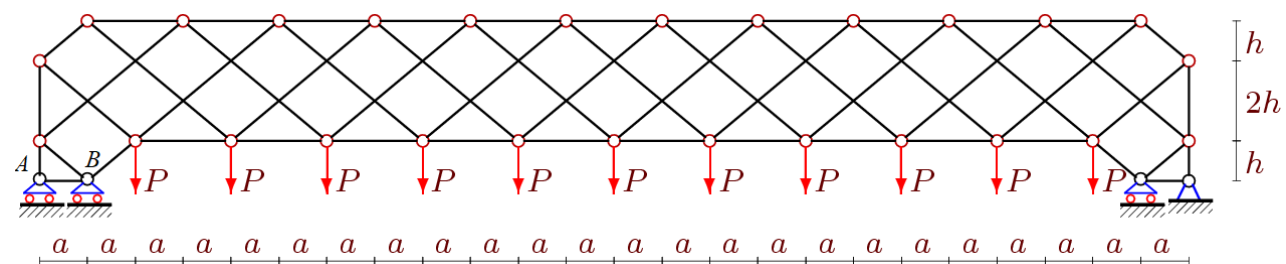

Fig. 1. Truss, load on the lower belt $n=5$

The procedure for calculating the forces in the core of a truss according to a program written in Maple is described in [7-9]. Data entry into the program begins with the location of the nodes. The fragment of the program with respect to the calculated truss has the form

$>$ for $i$ to 2 do

$>\mathbf{x}[i]:=a * i-a: y[i]:=0$ :

$>x[i+2 * n+3]:=a * i+2 *(2 * n+1) * a: y[i+2 * n+3]:=0:$

$>x[i+2 * n+5]:=0:$

$y[i+2 * n+5]:=2 * h * i-h:$

$>x[i+4 * n+9]:=2 * a *(2 * n+2):$

$y[i+4 * n+9]:=-2 * h * i+5 * h$ :

$>$ end:

$>$ for $i$ to $2 * n+1$ do

$x[i+2]:=2 * a * i: y[i+2]:=h:$

end:

$>$ for $i$ to $2 * n+2$ do

$x[i+2 * n+7]:=2 * a * i-a: y[i+2 * n+7]:=4 * h:$

end:

The matrix $G$ of a system of linear equations for the equilibrium of nodes is composed of cosine-directing forces in the rods.

From the solution of the matrix equation $G \bar{S}=\bar{B}$, where $\bar{S}$ - is the vector of all forces in the rods, $\bar{B}$ - the load vector, the forces are obtained in the symbolic form. The displacement of the middle node of the lower belt is determined by the Maxwell-Mohr's integral

$$
\Delta=\sum_{i=1}^{m-5} S_{i}^{(P)} S_{i}^{(1)} l_{i} /(E F) .
$$

It is indicated: $l_{i}$ - the length of the rods, $S_{i}^{(P)}$ - the forces in the rods under the given load, $S_{i}^{(1)}$ - the forces from the unit force applied to the knot of the lower belt in the middle of the span, $E F$ - the stiffnesses of the rods. The stiffnesses of all rods are the same.

\section{Results}

The first trial calculations in symbolic form according to the compiled program showed that the truss has a well hidden fault. This is manifested in the fact that for some values of $n$ the determinant of the system becomes zero. This corresponds to the kinematic degeneration of the truss. The truss becomes a mechanism that can be changed instantaneously, but this is enough to make it unusable as a supporting element. The calculations gave the following unacceptable values for the number $n: 1,4,7,10,13$. In order to exclude inadmissible 
values from the inductive method, the following function of the natural argument was introduced

$$
n=\left(1-(-1)^{k}+6 k\right) / 4 \text {. }
$$

The solution for trusses with an arbitrary number $k$ has the form

$$
\Delta=P\left(C_{1} a^{3}+C_{2} c^{3}+C_{3} h^{3}\right) /\left(2 h^{2} E F\right)
$$

The calculation of a series of trusses reveals sequences of coefficients for $a^{3}, h^{3}, c^{3}$.

The sequence of coefficients c $C_{1}$ is difficult to analyze: $94,161,1312,1637,6110$, 7021, 18376, 20345, 43438, 47081, 88064, 94141, 160462, 169877, 270280, 284081, 428606,447985 . The operator of the Maple system rgf_findrecur finds a recurrent equation of the ninth order that is satisfied by the terms of this sequence

$$
C_{1, k}=C_{1, k-1}+4 C_{1, k-2}-4 C_{1, k-3}-6 C_{1, k-4}-6 C_{1, k-5}+4 C_{1, k-6}-4 C_{1, k-7}-C_{1, k-8}+C_{1, k-9} .
$$

The solution of the equation is given by rsolve:

$$
\begin{aligned}
C_{1}= & \left(30 k^{4}+24\left(5-2(-1)^{k}\right) k^{3}+2\left(93-71(-1)^{k}\right) k^{2}+\right. \\
& \left.+4\left(25-19(-1)^{k}\right) k-21(-1)^{k}+29\right) / 8 .
\end{aligned}
$$

Similarly, from the equations

$$
\begin{gathered}
C_{2, k}=C_{2, k-1}+2 C_{2, k-2}-2 C_{2, k-3}-C_{2, k-4}+C_{2, k-5}, \\
C_{3, k}=2 C_{3, k-2}-C_{3, k-4} .
\end{gathered}
$$

we find other coefficients:

$$
\begin{gathered}
C_{2}=\left(12 k^{2}+2\left(12-7(-1)^{k}\right) k-5(-1)^{k}+13\right) / 4, \\
C_{3}=\left(3\left(9-5(-1)^{k}\right) k-8(-1)^{k}+12\right) / 2 .
\end{gathered}
$$

The algorithm used to solve the problem makes it easy to switch the task to another load. For this it is sufficient to introduce other coefficients in the vector $\bar{B}$ on the righthand side of the system. The loading of the upper belt in the Maple system (Fig. 2) has the form

$>$ for $i$ from $2 * n+8$ to $4 *_{n+9}$ do $B[2 * i]:=1:$ end:

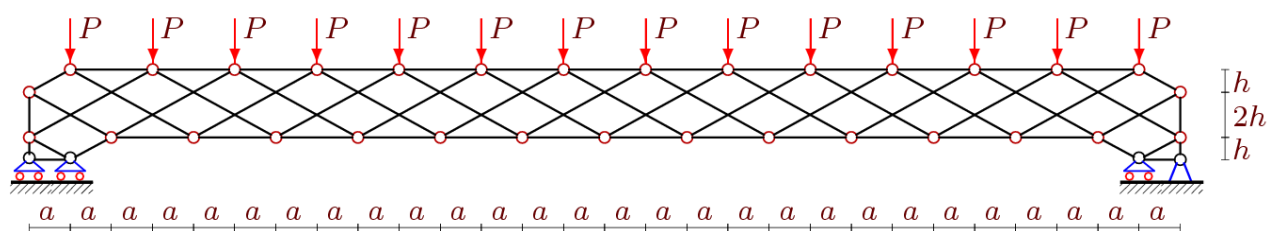

Fig. 2. Truss, load on the upper belt $n=6$

The solution has in this case the same form (1), but with the coefficients:

$$
\begin{gathered}
C_{1}=\left(30 k^{4}+24\left(5-2(-1)^{k}\right) k^{3}+6\left(35-23(-1)^{k}\right) k^{2}+4\left(33-25(-1)^{k}\right) k+11\left(1-(-1)^{k}\right)\right) / 8, \\
C_{2}=\left(6 k^{2}+\left(9-7(-1)^{k}\right) k-6(-1)^{k}+4\right) / 2, \\
C_{3}=\left(3\left(9-5(-1)^{k}\right) k-17(-1)^{k}+25\right) / 2 .
\end{gathered}
$$

We note that the recurrent equations remain the same. 
The simpler coefficients in (1) will be in calculating the deflection from one force in the middle of the lower belt (Fig. 3):

$$
\begin{gathered}
C_{1}=2 k^{3}+2\left(3-2(-1)^{k}\right) k^{2}+\left(11-8(-1)^{k}\right) k-(-1)^{k}+2, \\
C_{2}=\left(17+18 k-9(-1)^{k}\right) / 4, \\
C_{3}=\left(13-9(-1)^{k}\right) / 2 .
\end{gathered}
$$

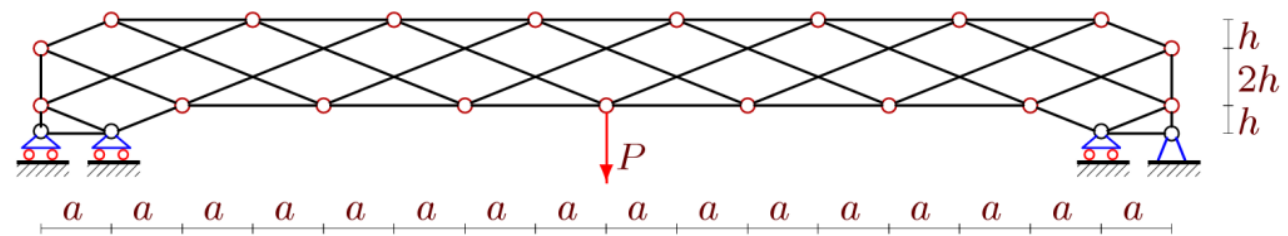

Fig. 3. Truss, load on the center, $n=3$

The equations for their definition are also simpler and have a smaller order:

$$
\begin{gathered}
C_{1, k}=C_{1, k-1}+3 C_{1, k-2}-3 C_{1, k-3}-3 C_{1, k-4}+3 C_{1, k-5}+C_{1, k-6}-C_{1, k-7}, \\
C_{2, k}=C_{2, k-1}+C_{2, k-2}-C_{2, k-3}, C_{3, k}=C_{3, k-2} .
\end{gathered}
$$

\section{Discussion}

The main value of the obtained solution is the obvious dependence of the deflection on the number of panels.

The main value of the obtained solution is the obvious dependence of the deflection on the number of panels. This can be demonstrated graphically. Suppose that the length of the span is fixed and does not depend on the number of panels $L=4 a(n+1)=100 \mathrm{~m}$. Then, when the number of panels is changed, the value of $a$ changes too. Also fix the total load $P_{0}$, regardless of the number of panels $P_{0}=P(2 n+1)$. We introduce a dimensionless amount of deflection, referring it to the load and the span length: $\Delta^{\prime}=\Delta E F /\left(P_{0} L\right)$. Figure 4 shows the curves plotted for the three heights of the truss.

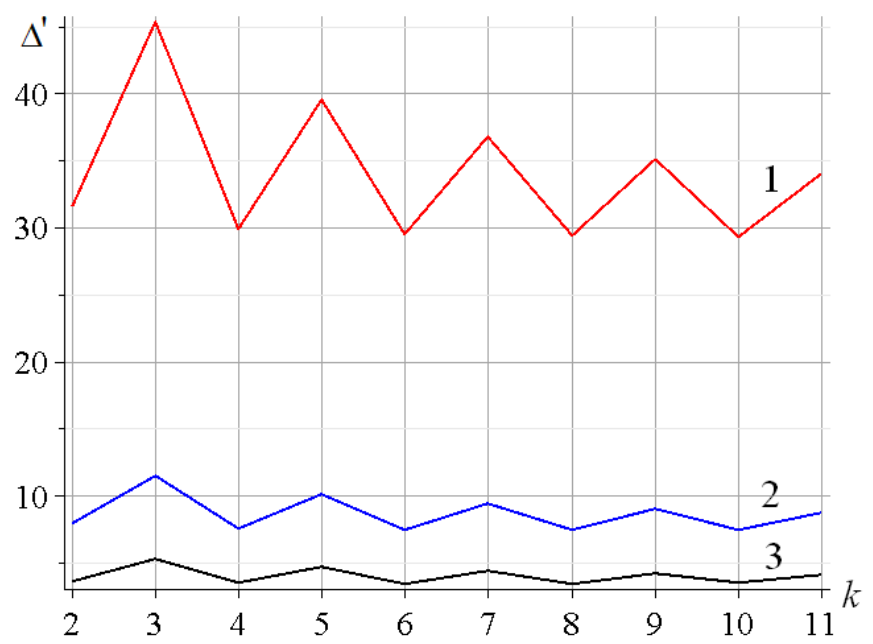

Fig 4. Dependence of the dimensionless deflection on the number of panels. Case of loading on the lower belt. $1-h=1.00 \mathrm{~m}, 2-h=2.00 \mathrm{~m}, 3-h=3.00 \mathrm{~m}$ 
First, there are striking strong jumps of curves. With an increase in the number of panels, the deflection jumps decrease. The very same jumps can be used to optimize the design due to the correct choice of the number of panels. It is easy to see that in some cases the change in the number of panels by just one can significantly change the deflection. The length of the span and the total loads remain unchanged.

In addition, attention is drawn to the strong nonlinear dependence of deflection on the height of the truss. Let us construct a graph of this dependence under the same assumptions about the span and the load for the case of loading the lower belt.

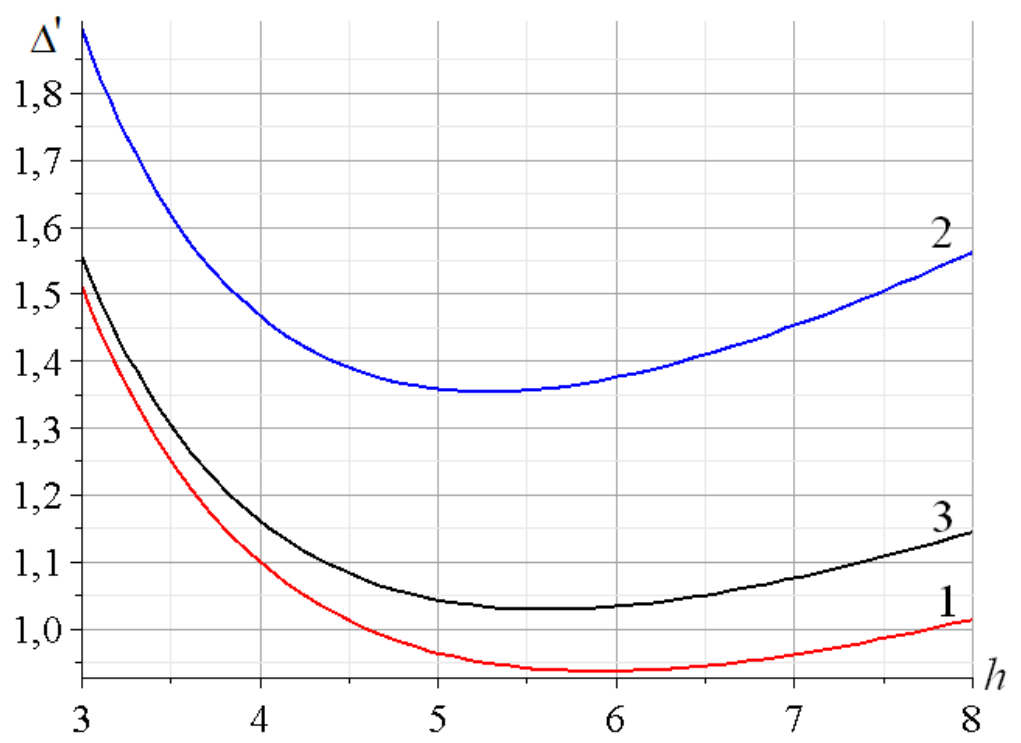

Fig 5. Dependence of the dimensionless deflection on the height of the truss under load on the lower belt, $L=60 \mathrm{~m} .1-k=10 ; 2-k=11 ; 3-k=12$

All curves have weakly expressed minima and with increasing height they will be reduced to an asymptote whose slope angle follows from the limit calculated by the operator limit (DEL/h, h=infinity) of the Maple system:

$$
\lim _{h \rightarrow \infty} \frac{\Delta^{\prime}}{h}=\frac{12 k^{2}+2\left(39-22(-1)^{k}\right) k+37-21(-1)^{k}}{4 L\left(3+6 k-(-1)^{k}\right)} .
$$

In addition, the order of the disposition of the curves is clearly violated. It would be more logical if curve 1 must be higher than 2 and 3 . The almost complete coincidence of curves 1 and 3 for $h<3 \mathrm{~m}$ shows that regardless of the number $k$, which characterizes the number of panels (10 or 12) at this height, the deflection is the same.

\section{Conclusions}

A new scheme of a statically definable truss has been proposed and analyzed analytically. It is shown that with some numbers of panels the truss can be an instantly changeable mechanism. This forces one to be careful in the analysis of building structures by numerical methods, where due to inaccuracies this effect can be unnoticed. Indeed, it was verified that if we slightly change the coordinates of the nodes, then the effect of the degeneration of the determinant disappears. In addition, a strong discontinuous dependence of the deflection on 
the number of panels and a rather smooth dependence of the solution on the height of the truss were observed. The solution found can be used both for testing numerical solutions and for simple estimates of the deformability of the designed systems.

\section{References}

1. K.A. Egarmin, G.Yu. Sysoev, N.I. Vatin, M.V. Vrublevskaya, Construction of Unique Buildings and Structures 2, 34 (2015)

2. M. Y. Baranovskiy, V. A. Tarasov, Construction of Unique Buildings and Structures 7, 92 (2014)

3. A.V. Alekseytsev, N.S. Kurchenko, Magazine of Civil Engineering, 5, 3 (2017)

4. I.N. Serpik, A.V. Alekseytsev, P.Yu. Balabin, N.S. Kurchenk, Magazine of Civil Engineering, 8, 181 (2017)

5. T. Saknite, D. Serdjuks, V. Goremikins, L. Pakrastins, N.I. Vatin, Magazine of Civil Engineering, 4, 26 (2016)

6. M.N. Kirsanov, N. Zaborskaya, Magazine of Civil Engineering, 3, 61 (2017)

7. M.N. Kirsanov, Magazine of Civil Engineering, 1, 33 (2016)

8. M.N. Kirsanov, Magazine of Civil Engineering, 5, 58 (2015)

9. M.N. Kirsanov, Magazine of Civil Engineering, 4, 38 (2015)

10. M.N. Kirsanov, Magazine of Civil Engineering, 4, 52 (2016)

11. M.N. Kirsanov, Magazine of Civil Engineering, 8, 58 (2017)

12. M.N. Kirsanov, Magazine of Civil Engineering, 1, 32 (2015)

13. M.N. Kirsanov, Magazine of Civil Engineering, 3, 3 (2015)

14. D.V. Tinkov, Magazine of Civil Engineering, 1, 25 (2016)

15. D.V. Tinkov, Magazine of Civil Engineering, 5, 66 (2015)

16. A. Kaveh, V. R. Mahdavi, Structural Engineering and Mechanics 5, 847 (2015)

17. A. Kaveh, A. Zolghadr, Applied Soft Computing Journal, 5, 2727 (2013)

18. A. Ahrari, D. Kalyanmoy, Computers \& Structures 164, 127 (2016)

19. I.N. Serpik, A.V. Alekseytsev, Magazine of Civil Engineering, 1, 14 (2016) 